\title{
Bombacoxylon owenii (Carr.) Gottwald from Gebel Shabraweet, Eastern Desert, Egypt
}

\author{
Marwah M. Kamal El-Din \\ Botany Department, Faculty of Science, \\ Ain Shams University, Cairo-Egypt. \\ E-mail:awwa_kamal@hotmail.com
}

\begin{abstract}
Kamal El-Din M. M. 2002. Bombacoxylon owenii (Carr.) Gottwald from Gebel Shabraweet, Eastern Desert, Egypt. Taeckholmia 22 (1): 91-99

Bombacoxylon owenii (Carr.) Gottwald (Bombacaceae), was widespread in Egypt in ages ranging from Oligocene to Quaternary. This is the first record of this species from Gebel Shabraweet. Comments on the distribution of all species of Bombacoxylon in the world are given.
\end{abstract}

Key words : Bombacoxylon, fossil wood, Gebel Shabraweet, Oligocene, wood anatomy.

\section{Introduction}

Bombacoxylon owenii had wide distribution in Egypt. It had been reported from 23 sites (Kräusel \& Stromer, 1924; Kräusel, 1939; Youssef, 1993; Kamal El-Din, 1996). Most of these sites exist in the Western Desert and a few in the Eastern Desert. It has a long vertical extension from Oligocene to Quaternary (cf. Kräusel, 1939).

This is the first record of fossil wood (Bombacoxylon owenii) from Gebel Shabraweet, which lies in the northern part of the Eastern Desert (latitude $30^{\circ} 18^{`}$ and longitude $32^{\circ} 17^{`}$ ), about $5 \mathrm{~km}$ to the south of Fayid city (Fig. 1). It is an Oligocene site.

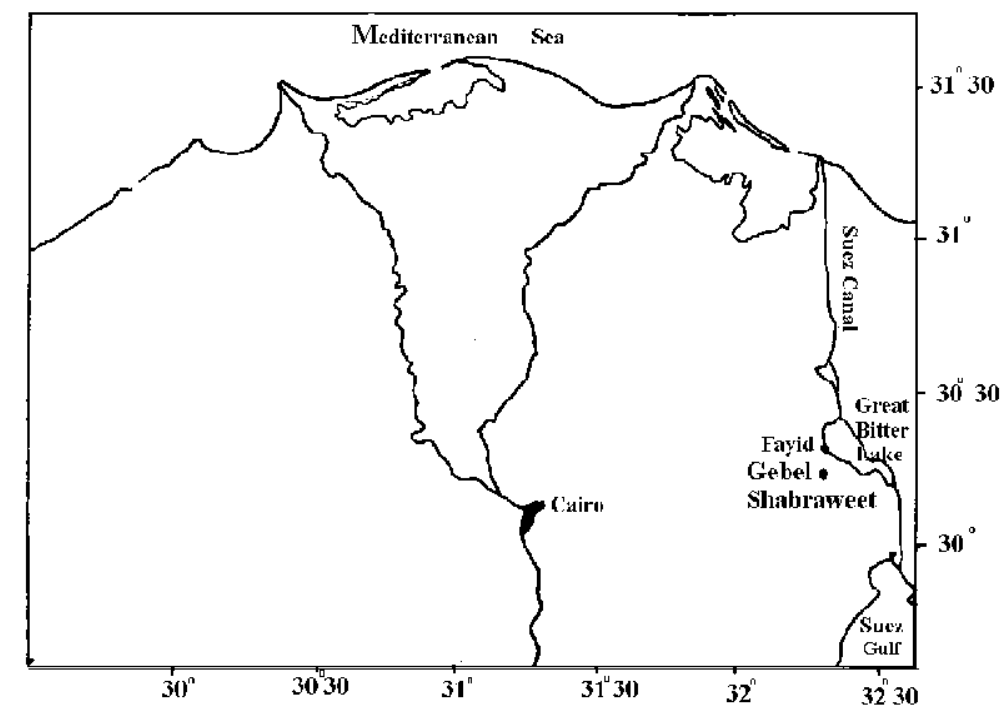

Fig. 1: Map showing location of Gebel Shabraweet (study area).

Received 5 June, 2002. Revision accepted 19 June, 2002. 
The aim of this paper is to provide a detailed description of the wood structure of the new specimens of Bombacoxylon owenii and to compare it with earlier descriptions from other sites in Egypt and other parts of the world. It aims also to discuss the global distribution of genus Bombacoxylon.

\section{Materials and Methods}

Two loose hand specimens were collected in 1997 from Gebel Shabraweet. The two specimens are about $17-20 \mathrm{~cm}$ in length and $6-8 \mathrm{~cm}$ in diameter. Thin ground cross, tangential and radial sections were prepared according to the method described by Lacey (1963). The specimens and the prepared slides are numbered and deposited in the palaeobotanical collection of the Department of Botany, Ain Shams University. Careful study showed that the two specimens belong to Bombacoxylon owenii.

\section{Description}

The following description of $B$. owenii is in accordance with the formate of IAWA list of the features suitable for hardwood identification (IAWA Committee, 1989).

Family : Bombacaceae

Genus : Bombacoxylon Gottwald 1969

Species : B. owenii $\quad$ (Carruthers) Gottwald 1969

\section{B. owenii (Figs. 2 - 6 )}

Growth rings boundaries intermediate between distinct and indistinct. Wood diffuse to semi-ring-porous. Vessels large, solitary or in radial multiples of 2-4 ( mostly in pairs), elliptic in shape, in diagonal pattern. Tangential diameter 200-260 $\mu \mathrm{m}$ ( mean $220 \mu \mathrm{m}$ ), radial diameter 330-420 $\mu \mathrm{m}$ (mean $350 \mu \mathrm{m}$ ). Vessels per sq. mm 2-5. Perforation plates simple with horizontal to oblique end walls. Intervessel pits alternate. Mean vessel element length $430 \mu \mathrm{m}$. Tyloses present.

Axial parenchyma mainly apotracheal diffuse in aggregates and paratracheal scanty to vasicentric but with narrow sheath. In LS parenchyma occur in strands.

Rays biseriate and a few uniseriate, 14-25 cells in height, 330-450 $\mu \mathrm{m}$ in length (mean $400 \mu \mathrm{m}$ ), mostly ended by a row of 2-3 cells, storied, homogenous with procumbent cells. Tile cells present (pterospermum type).

Fibers with simple to minutely bordered pits, nonseptate and have very thick walls. 


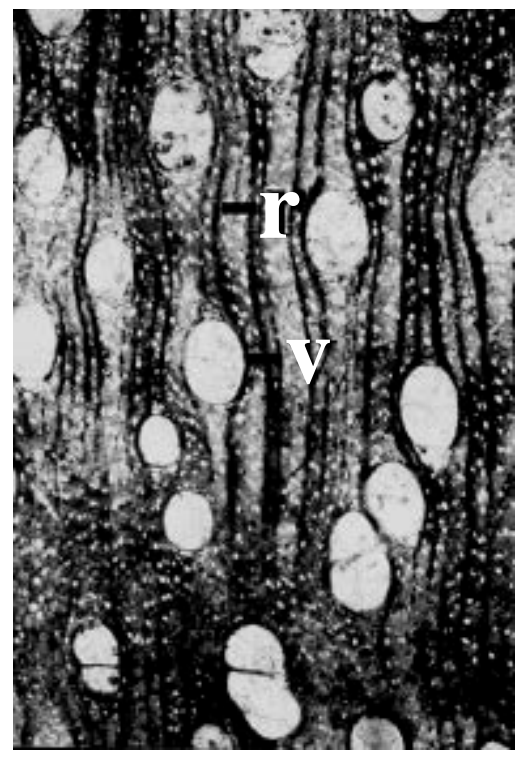

Fig. 2: Cross-section of Bombacoxylon owenii showing diffuse to semi-ringporous wood, solitary vessels, paired vessels and diffuse parenchyma, $\mathrm{x}$ 30. ( $\mathrm{v}=$ vessel, $\mathrm{r}=$ ray $)$.

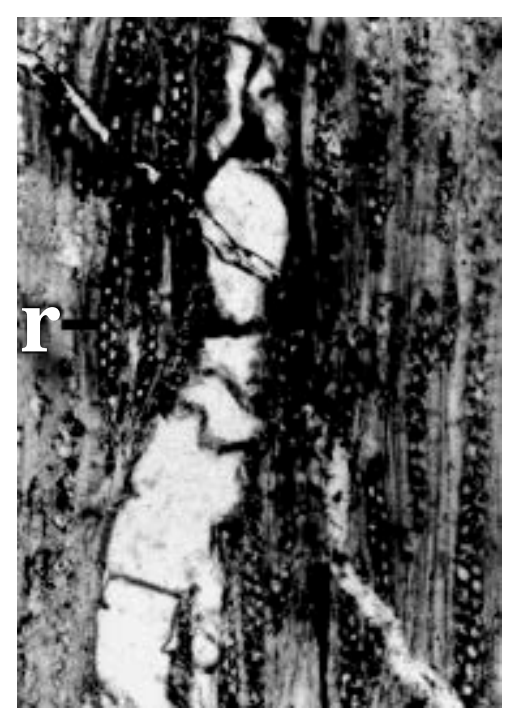

Fig. 4: Tangential-section of Bombacoxylon owenii showing mainly biseriate rays, $\mathrm{x} 50 .(\mathrm{r}=$ ray).

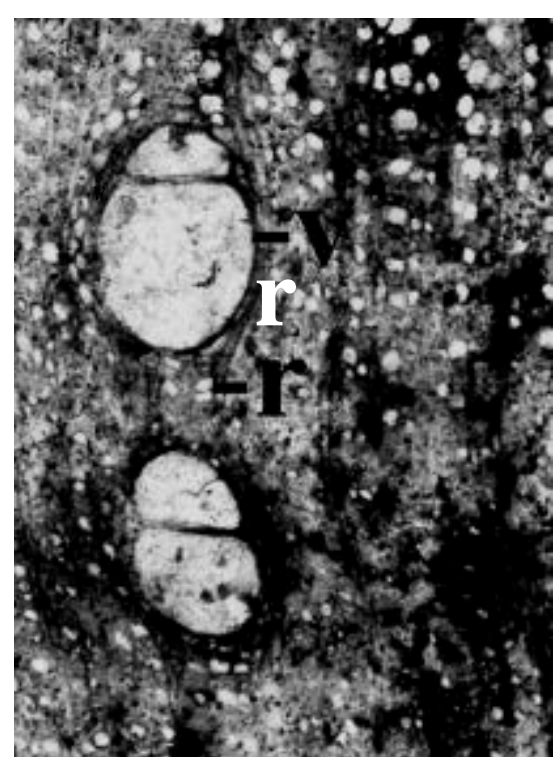

Fig. 3: Cross-section of Bombacoxylon owenii magnified to show scanty paratracheal and aggregates of diffuse apotracheal parenchyma, rays, paired vessels and tyloses, $\mathrm{x} 65$. ( $\mathrm{v}=$ vessel, $\mathrm{r}=$ ray)

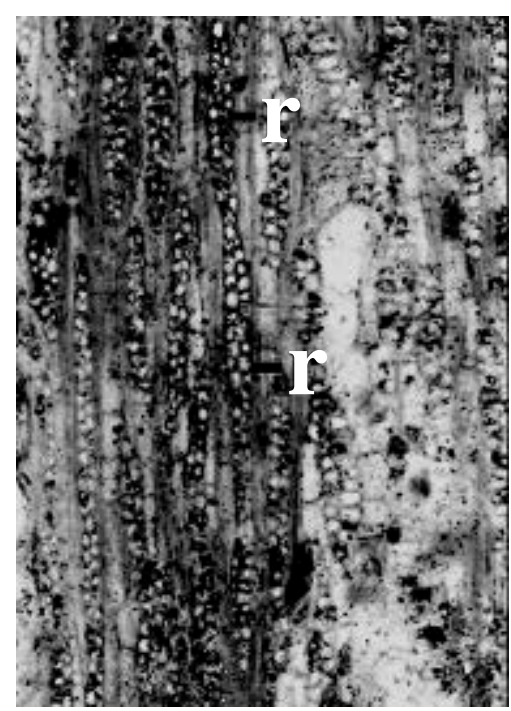

Fig. 5: Tangential- section of Bombacoxylon owenii magnified to show storied biseriate rays, $\mathrm{x} 50$. ( $\mathrm{r}=$ ray) 


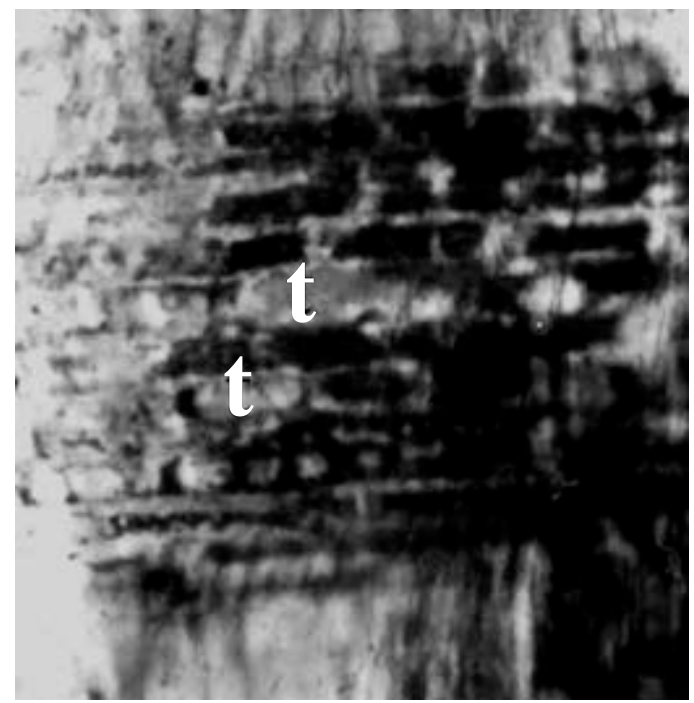

Fig. 6: Radial-section of Bombacoxylon owenii magnified magnified to show procumbent cells and tile cells ( $\mathrm{t}=$ tile cell $), \mathrm{x} 100$.

\section{Comparisons and Discussion}

Table 1a. Wood structure of $B$. owenii from Gebel Shabraweet is compared here with that described from other mainly Tertiary sites in Egypt, as given below.

\begin{tabular}{|c|c|c|c|c|}
\hline \multirow[b]{2}{*}{ Features } & \multicolumn{4}{|c|}{ Sites, ages and reference } \\
\hline & $\begin{array}{l}21 \text { sites in Egypt, } \\
\text { Tertiary and } \\
\text { Quaternary, } \\
\text { Kräusel (1939) }\end{array}$ & $\begin{array}{l}\text { West of Giza } \\
\text { Pyramids, } \\
\text { Miocene, } \\
\text { Youssef, (1993) }\end{array}$ & $\begin{array}{l}\text { Gebel El-Khashab } \\
\text { (southern forest), } \\
\text { Miocene, Kamal El- } \\
\text { Din (1996) }\end{array}$ & $\begin{array}{c}\text { Gebel } \\
\text { Shabraweet, } \\
\text { Oligocene, } \\
\text { Present work }\end{array}$ \\
\hline $\begin{array}{c}\text { Mean } \\
\text { tangential } \\
\text { diameter }\end{array}$ & $200 \mu \mathrm{m}$ & $260 \mu \mathrm{m}$ & $240 \mu \mathrm{m}$ & $220 \mu \mathrm{m}$ \\
\hline $\begin{array}{c}\text { Mean radial } \\
\text { diameter }\end{array}$ & $300 \mu \mathrm{m}$ & $410 \mu \mathrm{m}$ & - & $350 \mu \mathrm{m}$ \\
\hline $\begin{array}{l}\text { Mean vessel } \\
\text { element } \\
\text { length }\end{array}$ & $400 \mu \mathrm{m}$ & - & $500 \mu \mathrm{m}$ & $430 \mu \mathrm{m}$ \\
\hline $\begin{array}{l}\text { Vessels per } \\
\text { sq. mm }\end{array}$ & 1-6 (mostly 2-3) & - & $4-8$ & $2-5$ \\
\hline Rays & $\begin{array}{l}\text { Up to } 40 \text { (mostly } \\
10-20) \text { cells in } \\
\text { height }\end{array}$ & $\begin{array}{l}\text { 6-20 cells in } \\
\text { height }\end{array}$ & $\begin{array}{c}\text { Up to } 35 \text { cells in } \\
\text { height }\end{array}$ & $\begin{array}{l}\text { 14-25 cells in } \\
\text { height }\end{array}$ \\
\hline
\end{tabular}

Table 1a shows that the characteristic features of $B$. owenii from Gebel Shabraweet are slightly different from those of other parts of Egypt and the closest description to it is that given by Kräusel (1939). It must be mentioned, however that the description of $B$. owenii by Kräusel (1939) is apparently based on the study of specimens collected from 21 sites in Egypt ranging in age from Oligocene to Quaternary. 
Bombacoxylon owenii (Carr.) Gottwald from Gebel Shabraweet, Eastern Desert, Egypt

Specimens of $B$. owenii from Gebel Shabraweet are further compared with specimens described from other parts of the world (Table 1b).

Table 1b. Anatomical features of $B$. owenii from different ages and different countries of the world.

\begin{tabular}{|c|c|c|c|c|c|}
\hline \multirow[b]{2}{*}{ Features } & \multicolumn{5}{|c|}{ Sites, ages and reference } \\
\hline & $\begin{array}{c}\text { Sahara West } \\
\text { of Tunisia, } \\
\text { Oligocene, } \\
\text { Gottwald } \\
\text { (1969) }\end{array}$ & $\begin{array}{c}\text { Algeria, } \\
\text { Neogene, } \\
\text { Koeniguer } \\
\text { (1972) }\end{array}$ & $\begin{array}{c}\text { Ethiopia, } \\
\text { terminal } \\
\text { Cretaceous- } \\
\text { Eocene, } \\
\text { Beauchamp \& } \\
\text { Lemoigne } \\
\text { (1973b) }\end{array}$ & $\begin{array}{l}\text { France (Bas- } \\
\text { et-Lezat), } \\
\text { Oligocene, } \\
\text { Privé-Gill \& } \\
\text { Pelletier } \\
\text { (1981) }\end{array}$ & $\begin{array}{c}\text { Egypt (Gebel } \\
\text { Shabraweet), } \\
\text { Oligocene, } \\
\text { this work }\end{array}$ \\
\hline $\begin{array}{l}\text { Mean tangential } \\
\text { diameter }\end{array}$ & $200 \mu \mathrm{m}$ & $\begin{array}{c}130-220 \\
\mu \mathrm{m}\end{array}$ & $250 \mu \mathrm{m}$ & $200 \mu \mathrm{m}$ & $220 \mu \mathrm{m}$ \\
\hline $\begin{array}{l}\text { Mean vessel } \\
\text { element length }\end{array}$ & $425 \mu \mathrm{m}$ & - & - & - & $430 \mu \mathrm{m}$ \\
\hline $\begin{array}{c}\text { Vessels per sq. } \\
\text { mm }\end{array}$ & - & $5-6$ & $3-5$ & $3-5$ & $2-5$ \\
\hline Rays & $\begin{array}{l}\text { 6-28 cells in } \\
\text { height }\end{array}$ & - & 1-4 seriate & $\begin{array}{l}2-19 \text { cells in } \\
\text { height }\end{array}$ & $\begin{array}{l}\text { 14-25 cells in } \\
\text { height }\end{array}$ \\
\hline
\end{tabular}

It will be noticed that, $B$. owenii specimens described by Gottwald (1969) from Tunisia are the nearest in anatomical features to specimens from Gebel Shabraweet, even nearer than description given by Kräusel (1939).

The geologic age of $B$. owenii in Egypt ranges from Oligocene to Quaternary. However, it seems to have been most widespread in the Miocene since the number of sites was largest during that age compared to earlier and later ages (Fig. 7): Oligocene (7 sites; Qattamiya, Mokattam, Gebel Ahmer, Bir El-Fahme, Turra, Wadi Ankebieh and Fayum), Oligocene-Miocene (3 sites; Bahariya, Giza Pyramids and Gebel El-Khashab "northern petrified forest" ), Miocene (10 sites; Wadi Faregh, Garet Aujan, Dêr Baramûs, Bir Lebuk, Moghara, Wadi El-Natrun, Gebel Geneffe, Suez, west of Giza Pyramids and Gebel ElKhashab "southern petrified forest"), Pliocene (2 sites; Gebel Ahmer and Wadi Sanur) and Quaternary (one site; Birket Qerûn).

Specimens of more or less similar age (Tertiary and Quaternary) are known also from Asia (Jordan or Israel, Pakistan), Europe (France, Sardinia) and Africa (Algeria, Ethiopia, Libya, Rio de Oro, Somalia and Tunisia). However, in Africa (Ethiopia) specimens as early as terminal Cretaceous-Eocene have been reported (Kräusel, 1939; Koeniguer, 1966, 1967, 1972; Gottwald, 1969; Beauchamp \& Lemoigne, 1973 a, b; Beauchamp et al., 1973; Louvet, 1973; Privé-Gill \& Pelletier, 1981; Boureau et al., 1983; Dupéron-Laudoueneix \& Dupéron, 1995; Dupéron et al., 1996)

Genus Bombacoxylon comprises seven species, which are known from Africa except $B$. owenii which exists in Asia and Europe besides Africa as well as B. langstoni which has been discovered recently (Wheeler \& Lehman, 2000) from USA as shown in Table 2.

Table 2 shows that four species of Bombacoxylon occur in Ethiopia, justifying the idea that Ethiopia (Africa) may be the center of evolution of Bombacoxylon, especially that Bombacoxylon had a much longer vertical extension in that continent. 


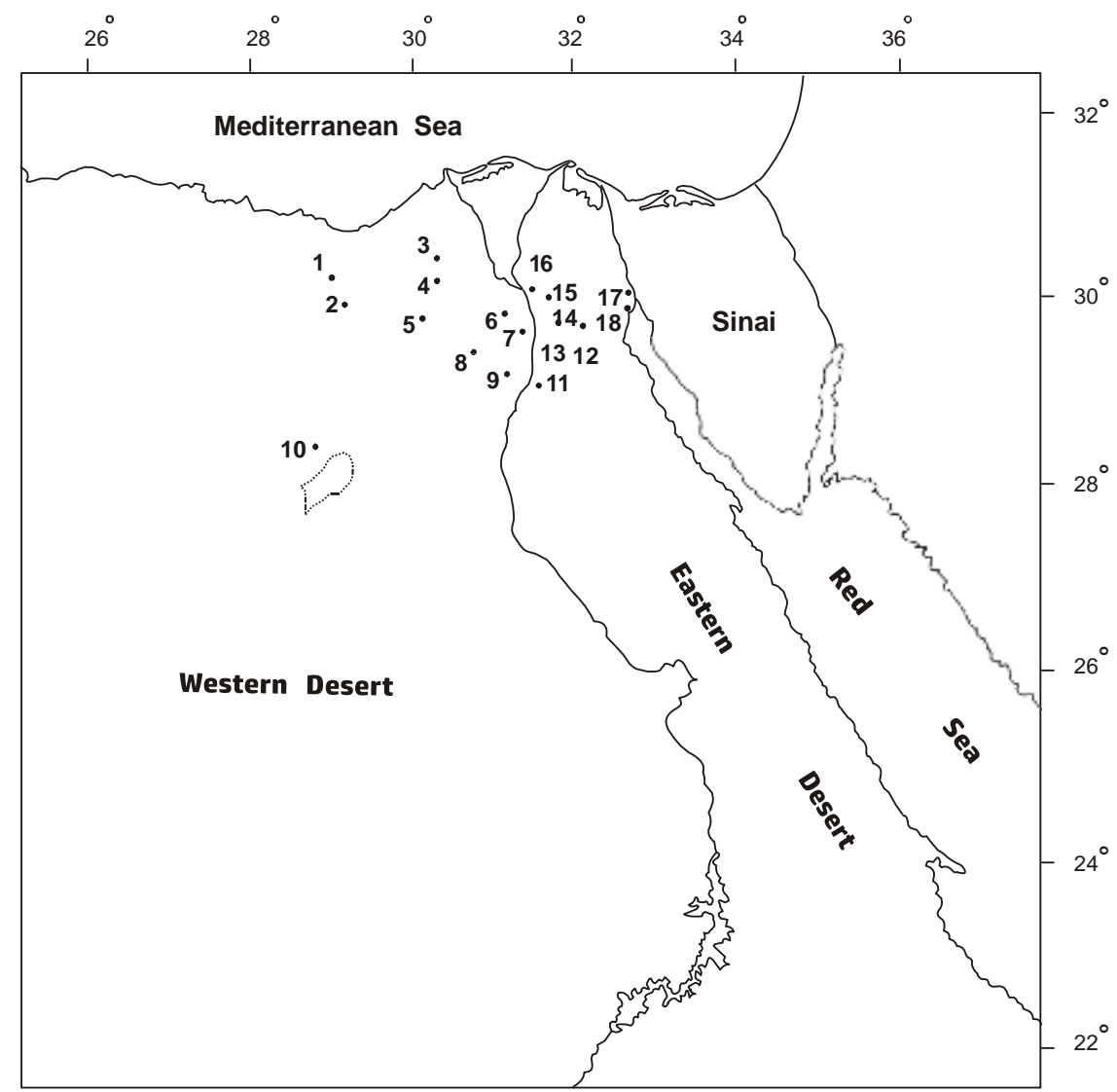

Fig. 7. Map showing the sites of Bombacoxylon owenii reported from Egypt, based on Kräusel (1939), Youssef (1993) \& Kamal El-Din (1996). 1- Bir Lebuk, 2- Moghara, 3- Wadi El-Natrun, 4- Dêr Baramûs, 5- Wadi Faregh and Garet Aujan, 6- Gebel El-Khashab (southern and northern petrified forest), 7- Giza Pyramids and west of Giza Pyramids, 8- Birket Qerûn, 9- Fayum, 10Bahariya, 11- Wadi Sanur, 12- Wadi Ankebieh, 13- Qattamiya, 14- Mokattam, 15- Gebel Ahmer and Bir El- Fahme, 16- Turra, 17- Gebel Geneffe, 18- Suez.

Table 2 also shows that $B$. owenii is the most widespread species of Bombacoxylon being represented in Asia, Europe and Africa, where it was reported from seven countries. Further more B. owenii was the most common tree in the Oligocene forests of Egypt. Thus Kamal El-Din (1996) reported 39 well preserved dicot tree trunks from the site of the petrified forest at Gebel El-Khashab, 21 out of which were found to belong to Bombacoxylon owenii (Fig. 8) and the rest (18) were related to 7 other species. 
Table 2. Distribution of the seven known Bombacoxylon species in different countries of the world:

\begin{tabular}{|c|c|c|c|}
\hline Species name & Age & Country & Reference \\
\hline $\begin{array}{l}\text { 1- B. affine (Felix) } \\
\text { Gottwald }\end{array}$ & Tertiary & Ethiopia & $\begin{array}{l}\text { Felix, 1887; Gottwald, 1969; } \\
\text { Privé-Gill \& Pelletier, 1981; } \\
\text { Dupéron- Laudoueneix \& } \\
\text { Dupéron, } 1995\end{array}$ \\
\hline $\begin{array}{l}\text { 2- B. bombacoides } \\
\text { (Bancroft) } \\
\text { Kräusel }\end{array}$ & Miocene & Kenya & $\begin{array}{l}\text { Bancroft, 1932; Kräusel, 1939; } \\
\text { Privé-Gill \& Pelletier, 1981; } \\
\text { Dupéron- Laudoueneix \& } \\
\text { Dupéron, } 1995\end{array}$ \\
\hline $\begin{array}{l}\text { 3- B. gallettii } \\
\text { Beauchamp \& } \\
\text { Lemoigne }\end{array}$ & $\begin{array}{l}\text { terminal } \\
\text { Cretaceous- } \\
\text { Eocene }\end{array}$ & Ethiopia & $\begin{array}{l}\text { Beauchamp \& Lemoigne, } 1973 \\
\text { a, b; Privé-Gill \& Pelletier, } \\
\text { 1981; Dupéron- Laudoueneix \& } \\
\text { Dupéron, } 1995\end{array}$ \\
\hline $\begin{array}{l}\text { 4- B. grambastii } \\
\text { Lemoigne }\end{array}$ & $\begin{array}{l}\text { Pliocene- } \\
\text { Quaternary }\end{array}$ & Ethiopia & $\begin{array}{l}\text { Lemoigne, 1978; Privé-Gill \& } \\
\text { Pelletier, 1981; Dupéron- } \\
\text { Laudoueneix \& Dupéron, } 1995\end{array}$ \\
\hline $\begin{array}{l}\text { 5- B. monodii } \\
\text { (Boureau) } \\
\text { Gottwald }\end{array}$ & Tertiary & $\begin{array}{l}\text { Algeria and } \\
\text { Mali } \\
\text { (Sudanese } \\
\text { Sahara) }\end{array}$ & $\begin{array}{l}\text { Boureau, 1949; Gottwald, 1969; } \\
\text { Privé-Gill \& Pelletier, 1981; } \\
\text { Dupéron- Laudoueneix \& } \\
\text { Dupéron, } 1995 .\end{array}$ \\
\hline $\begin{array}{l}\text { 6- B. owenii } \\
\text { (Carr.) } \\
\text { Gottwald }\end{array}$ & $\begin{array}{l}\text { terminal } \\
\text { Cretaceous- } \\
\text { Quaternary }\end{array}$ & $\begin{array}{l}\text { Jordan or } \\
\text { Israel, } \\
\text { Pakistan, } \\
\text { France, } \\
\text { Sardinia, } \\
\text { Algeria, } \\
\text { Egypt, } \\
\text { Ethiopia, } \\
\text { Libya, Rio de } \\
\text { Oro, Somalia } \\
\text { \& Tunisia } \\
\end{array}$ & $\begin{array}{l}\text { Kräusel \& Stromer, 1924; } \\
\text { Kräusel, 1939; Koeniguer, } \\
\text { 1966, 1967, 1972, Gottwald, } \\
\text { 1969; Beauchamp \& Lemoigne, } \\
\text { 1973 a, b; Beauchamp et al., } \\
\text { 1973; Louvet, 1973; Privé-Gill } \\
\text { \& Pelletier, 1981; Boureau et } \\
\text { al., 1983; Youssef, 1993; } \\
\text { Dupéron- Laudoueneix \& } \\
\text { Dupéron, 1995; Dupéron et al. , } \\
\text { 1996; Kamal El-Din, 1996. }\end{array}$ \\
\hline $\begin{array}{l}\text { 7- B. langstoni } \\
\text { Wheeler \& } \\
\text { Lehman } \\
\end{array}$ & $\begin{array}{l}\text { Late } \\
\text { Cretaceous }\end{array}$ & $\overline{\text { USA }}$ & Wheeler \& Lehman, 2000 \\
\hline
\end{tabular}




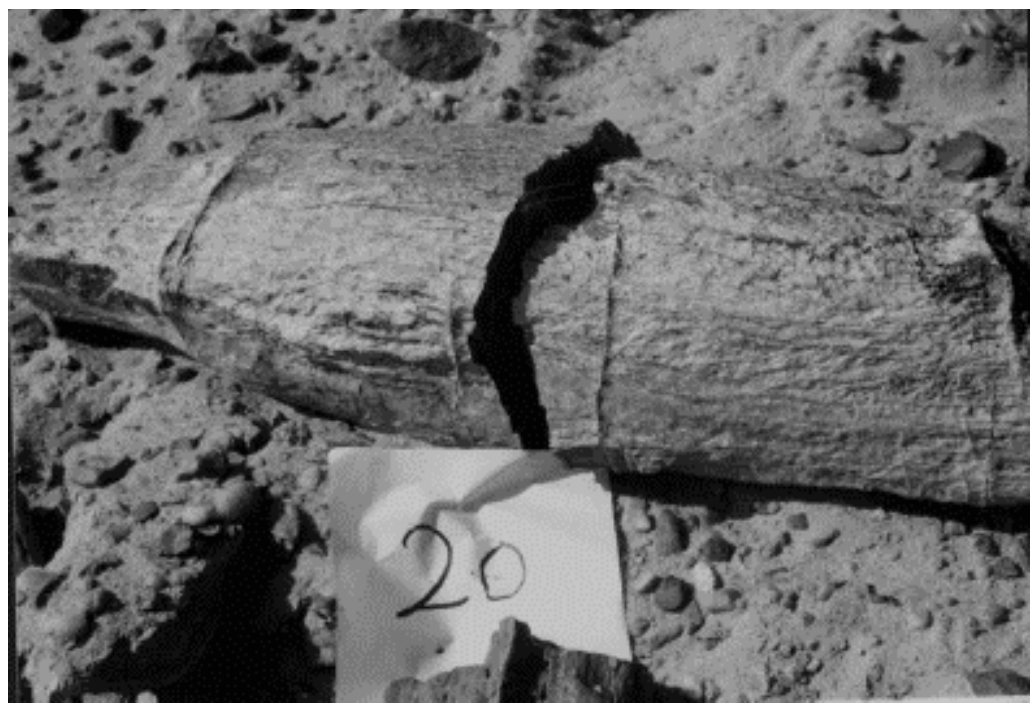

Fig. 8. One of the 21 Bombacoxylon owenii trunks found at the site of the southern petrified forest at Gebel El-Khashab.

In addition, an eighth species of Bombacoxylon reported by Kamal El-Din (1996) from Gebel El-Khashab, and believed to be new to science, will be the subject of a future publication.

\section{Acknowledgements}

Thanks to Dr. Wagieh El-Saadawi, Professor of Botany at Faculty of Science, Ain Shams University for useful criticism and for continuous help. Thanks are also due to Dr. Osman Abd El-Ghany, Lecturer in Geology, Faculty of Science Ain Shams University, for providing the specimens which were the subject of this study.

\section{References}

Bancroft H. 1932. Some fossil Dicotyledonous woods from the Miocene (?) beds of East of Africa. Ann. Bot., 46: 745-467.

Beauchamp J. \& Lemoigne Y. 1973 a. Age du facies "grès supérieur" et du début du volcanismedes Trappes dans le Massif du Chercher(Province d’ Harar, Ethiopie). C. R. Acd. Sci. Paris D, 276 (10): 1525-1527.

-1973 b. Description d` unepaléoflore du Crétacé terminalEocène dans le Massif du Chercher (Province d’ Harar, Ethiopie). Doc. Lab. Géol. Fac. Sci. Lyon, 56: 167-179.

\& Petrescu J. 1973. Les paléoflore Tertiaires de Debré-Libanos (Ethiopie). Ann. Soc. Géol. Nord. 93 (1): 17-32. 
Bombacoxylon owenii (Carr.) Gottwald from Gebel Shabraweet, Eastern Desert, Egypt

Boureau E. 1949. Etude paléoxylologique du Sahara (V). Sur le Dombeyoxylon monodii n. sp., Sterculiacée fossile des environs de Tindouf et de L`Azaouad. Bull. Mus. Natl. Hist. Nat., 2e sér., 21 (5): 639-646.

-, Cheboldaeff-Salard M. „Koeniguer J. \& Louvet P. 1983. Evolution des flores et de la végétation Tertiaires en Afrique, au nord de l' Equateur. Bothalia 14, 3 \& 4: 355-367.

Dupéron J. , Dupéron-Laudoueneix M. \& Durrani K. H. 1996. Découverte de Bombacoxylon wenii (Carr.) Gott. Dan le Cénozoique du Pakistan. Historique et intérêts de L` espéce.: 59-75, 1-pl., 3- fig., 1-tab..

Dupéron-Laudoueneix M. \& Dupéron J. 1995. Inventory of Mesozoic and Cenozoic woods from Equatorial and North Equatorial Africa. Rev. of Palaeobot. and Palynol. 84: $439-480$.

Felix J. 1887. Beiträge zur kenntniss der fossilen Hölzer Ungarns. Mitt. Jahrb. Ungar. Geol. Anst., 8 (5): 145-162.

Gottwald H. 1969. Zwei kieseelhölzer aus dem Oligäzan von Tunis, Bombacoxylon owenii und Pseudolachnostyloxylon weylandii. Palaeontographica B., 125(4-6):112-118.

IAWA Committee 1989. IAWA list of microscopic features for hardwood identification. International Association of Wood Anatomists Bulletin 10:219-332 (New series).

Kamal El-Din M. M. 1996. Studies on a petrified wood at Gebel El-Khashab, Western Desert, Egypt. M. Sc. Thesis, Dept. of Bot.Fac. of Science, Ain Shams University.

Koeniguer J. 1966. Etude paléoxylogique de la Libya. II. Sur la présence de Dombeyoxylon owenii (Carr.) Kräusel, 1939, dans le Tertiaire de la Syrte. 91e Congr. Natl. Soc. Sav., Rennes, 1966, Sci., 3: 164-168. 1967. Etude paléoxylogique Du Rio de Oro. Not. Com. Inst. Geol. Min. Esp., 96: 39-66. -1972. Sur quelques bois fossiles du Tertiaire de l `Algérie. 97e Congr. Natl. Soc. Sav., Nantes, Sci., 4: 45-65 (1976).

Kräusel R. 1939. Ergebnisse der Forschungsreisen Prof. E. Stromers in den Wüsten Ägyptens. Abh. Bayer. Akad. Wiss., München 47: 1 - 140.

Kräusel R. \& Stromers E. 1924. Ergebnisse der Forschungsreisen Prof. E. Stromers in den Wüsten Ägyptens. Abh. Bayer. Akad. Wiss., München 30: 1 - 48.

Lacey, W.S. 1963. Palaeobotany Technique, J.D. Carthey and I. Duddington (eds.). View Point in Biology. Butterworths, London 2: 202 - 243.

Lemoigne Y. 1978. Flores tertiaires de la haute vallée de L`Omo (Ethiopie). Palaeontographica, B, 165 (4-6): 89-157.

Louvet P. 1973. Sur les affinités des flores tropicales ligneuses africaines tertiaire et actuelle. Bull. Soc. Bot. France. 120: 385-395.

Privé-Gill C. \& Pelletier H. 1981. Sur quelques bois silicifiés du Tertiaire de limagne, dans la region d`Aiguesperse (Puy-de-Dôme), France. Review of Palaeobot. and Palyno. 34: 369-405.

Wheeler E. A. \& Lehman T. M. 2000. Late Cretaceous woody dicots from the Aguja and Javelina Formation, Big Bend National Park, Texas, USA. IAWA J. 21(1):83-120.

Youssef S. G. 1993. Studies on some Egyptian fossil woods. Ph. D. Thesis, Dept. of Bot. Fac. of Science, Zagazig Uni. Benha. 